\title{
The Effect of Hyperbolic Two-temperatures Model on Waves Propagation in a Semi-conductor Medium Containing Spherical Cavities
}

Ibrahim Abbas ( $\square$ ibrabbas7@yahoo.com)

Department of mathematics, Faculty of Science, Sohag University, Sohag, Egypt

https://orcid.org/0000-0001-9595-439X

Aatef Hobiny

King Abdulaziz University Faculty of Sciences

\section{Research Article}

Keywords: Laplace transforms, spherical cavity, thermal relaxation time, hyperbolic two-temperature, eigenvalues approach

Posted Date: August 19th, 2021

DOI: https://doi.org/10.21203/rs.3.rs-815210/v1

License: (c) (i) This work is licensed under a Creative Commons Attribution 4.0 International License. Read Full License 


\title{
The effect of hyperbolic two-temperatures model on waves propagation in a semi-conductor medium containing spherical cavities
}

\author{
Ibrahim A. Abbas ${ }^{a, b} \&$ Aatef Hobiny \\ aDepartment of mathematics, Faculty of Science, Sohag University, Sohag, Egypt. \\ ${ }^{b}$ Department of Mathematics, Faculty of Science, King Abdulaziz University, Saudi Arabia \\ E-mail : ibrabbas7@yahoo.com \& ahobany@kau.edu.sa
}

\begin{abstract}
This article is interested in the study of the carrier density, the redial displacement, the conductive temperature, thermodynamic temperature and the stresses in a semi-conductor material containing a spherical hole. This investigation deals with the photo-thermo-elastic interactions in a semi-conductor medium in the context of the new hyperbolic two-temperatures model with one relaxation time. The Laplace transform technique are used to obtain the problem analytical solution by the eigenvalues methods and the inversions of the Laplace transform were performed numerically. Numerical results for semi-conductor materials are shown graphically and discussed.
\end{abstract}

Keywords: Laplace transforms; spherical cavity; thermal relaxation time; hyperbolic twotemperature; eigenvalues approach.

PACS: 52.38.Ph, 78.40.Fy, 72.20.Pa

\section{Nomenclature}

$\begin{array}{ll}T=T^{*}-T_{o}, T^{*} & \text { the variations of temperature } \\ T_{o} & \text { the reference temperature } \\ t & \text { the time } \\ u_{i} & \text { the displacement components } \\ \rho & \text { the density of material } \\ c_{e} & \text { the specific heat at constant strain, } \\ \tau & \text { the lifetime of photo-generated carrier, } \\ N=n-n_{o}, n_{o} & \text { the carrier concentration at equilibrium, } \\ \gamma_{n}=(3 \lambda+2 \mu) d_{n}, d_{n} & \text { the electronic deformation coefficient, }\end{array}$




\begin{tabular}{|c|c|}
\hline$k=\frac{\partial n_{o}}{\partial T}$ & the coupling parameter of thermal activation \\
\hline$K$ & the thermal conductivity \\
\hline$\gamma_{t}=(3 \lambda+2 \mu) \alpha_{t}, \alpha_{t}$ & the linear thermal expansion coefficients \\
\hline$\sigma_{i j}$ & the components of stresses, \\
\hline$D_{e}$ & the carrier diffusion coefficient, \\
\hline$\lambda, \mu$ & the Lame's constants, \\
\hline$T_{1}$ & the constant temperature \\
\hline$\tau_{o}$ & the thermal relaxation time \\
\hline$q_{o}$ & constant \\
\hline$t_{p}$ & the pulsing heat flux characteristic time \\
\hline$s_{b}$ & the speed of recombination on the surface \\
\hline$R$ & the internal redial of cavity \\
\hline
\end{tabular}

\section{Introduction}

The theory of thermoelasticity, which is the most common engineered structural material, plays an important role in steel stress analysis and applied mechanical science. It can describe the solid mechanical behavior of some common elastic materials like coal, concrete and wood. However, it cannot describe the mechanical behavior of many synthetic materials of polymer and clastomer type such as polyethylene. The temperature increment of body is not only caused by internal and external heat sources, but also by deformations of itself process during the micro-inertia of the microelement. In the first half of the last century, many authors used the theory of generalized thermoelasticity to describe elastic and thermal waves in elastic materials such as semiconductors (semi-insulating). In this case, semiconductor materials have been studied as an elastic support only. But at the end of the last century, various scientists studied semiconductor materials in particular their internal structures during microelectronic processes.

Biot [1] developed the coupled thermoelasticity theory (CD theory) when motived the law of Fourier heat conduction that became appropriate for modern engineering applications spicily in high temperature case. But in low temperature case, the thermoelastic models are physically unacceptable and cannot obtain equilibrium state. Lord et al. [2] (LS) inserted one relaxation time in the heat conduction equation (Fourier's law of heat conduction) to overcome this contradiction. The thermo-elasticity model with classical two-temperatures are presented by and Chen et al. [3], 
Chen and Gurtin [4] and Williams and Gurtin [5], by using another depending on the classic twotemperature (the conductivity temperature $\phi^{*}$ and the thermodynamically temperature $T^{*}$ ). Recently, Youssef et al. [6] investigated a new model in generalized thermoelsticity theory when they introduced the theory of hyperbolic two-temperature. Taye et al. [7] studied the hyperbolic two-temperature semiconductor thermoelastic wave by laser pulses. Saeed and Abbas [8] studied the hyperbolic two-temperatures photothermal interaction in a semi-conductor medium. Abbas et al. [9] discussed the hyperbolic two-temperature photothermal interactions in a semi-conductor material with a cylindrical cavity. Lotfy et al. [10] investigated the effect of variable thermal conductivity of a semiconducting medium with cavities under the fractional-order magnetophotothermal model. Lotfy et al. [11] investigated the response of Thomson and magnitic impact of semiconducor material due to laser pulses under photothermoelastic theory. Hobiny and Abbas [12] investigated the photothermal interaction in a two-dimension semi-conductor plane under the GN model. Ali et al. [13] studied the reflections of wave in a rotating semi-conductor nanostructure material through torsion-free boundary conditions. Yasein [14] discussed the influences of variable thermal conductivity of semi-conductor medium under photothermal model due to thermal ramp type. Lotfy et al. [15] discussed the Thomson and electromagnetic effects under the photo-thermal model of a rotator semiconductor materials with hydrostatic initial stress. Alzahrani and Abbas [16] studied the photo-thermoelastic interaction in a semi-conductor plane without energy dissipations. Abbas and Hobiny [17] used the finite differnce method to study the photothermal interraction in simecondactor medium. Youssef and El-Bary [18] studied the characterization of the photo-thermal interactions of a semiconductor solid sphere due to the fractional deformations, the thermal relaxation times, and various references temperature under L-S model. Lotfy et al [19] the photo-thermal excitations process during hyperbolic two-temperature model for magnetothermo-elastic semiconductor material. Hobiny and Abbas [20] investigated the photo-thermal wave in an infinite semi-conductor medium containing cylindrical hole. Many authors [21-36] solved several problems by using numerical and analytical approaches for thermal and elastic waves.

This work is devoted to an investigate of the analyticcal solutions of photothermal interaction in semi-conductor mediums with a spherical hole under the new hyperbolic of two-temperatures thermo-elasticity. The effect of the two-temperature parameter on the thermodynamic and the 
conductive temperatures, the stress, the redial displacement and the carrier density distributions have been depicted graphically.

\section{Basic equations}

In this article, theoretical dissuasions during the heat transport process when the internal structure of the semi-conductor is taken into consideration. The interactions between thermal and elastic waves of the plasma are generated in the context of the own temperature (both hyperbolic temperatures). The governing equations under photo-thermal model with the hyperbolic twotemperatures in semiconductor medium can be given by $[6,37,38]$ :

The equations of motion:

$\mu u_{i, j j}+(\lambda+\mu) u_{j, i j}-\gamma_{n} N_{, i}-\gamma_{t} T_{, i}=\rho \frac{\partial^{2} u_{i}}{\partial t^{2}}$.

The coupling between thermoelastic and plasma waves can be expressed as

$D_{e} N_{, j j}-\frac{N}{\tau}+\frac{k}{\tau} T=\frac{\partial N}{\partial t}$.

The equation of heat conduction

$K \phi_{, j j}+\frac{E_{g}}{\tau} N=\left(1+\tau_{o} \frac{\partial}{\partial t}\right)\left(\rho c_{e} \frac{\partial T}{\partial t}+\gamma_{t} T_{o} \frac{\partial u_{j, j}}{\partial t}\right)$.

The new hyperbolic of two-temperature relation

$\ddot{\phi}-\ddot{T}=a \phi, j j$.

The stress-strain relations are expressed as

$\sigma_{i j}=\mu\left(u_{i, j}+u_{j, i}\right)-\left(\gamma_{n} N+\gamma_{t} T-\lambda u_{k, k}\right) \delta_{i j}$,

Let us consider a homogeneous, isotropic unbounded semi-conductor medium containing a sphherical hole, whose state can be expressed in terms of the space variable $r$ and the time $t$ which occupying the region $R \leq r<\infty$. The radial displacement $u_{r}=u(r, t)$ non-vanishing only due to symmetry, hence the equations (1)-(5) can be rewritten by:

$(\lambda+2 \mu)\left(\frac{\partial^{2} u}{\partial r^{2}}+\frac{2}{r} \frac{\partial u}{\partial r}-\frac{u}{r^{2}}\right)-\gamma_{t} \frac{\partial \mathrm{T}}{\partial r}-\gamma_{n} \frac{\partial N}{\partial r}=\rho \frac{\partial^{2} u}{\partial t^{2}}$,

$D_{e}\left(\frac{\partial^{2} N}{\partial r^{2}}+\frac{2}{r} \frac{\partial N}{\partial r}\right)=\frac{\partial N}{\partial t}-\frac{k}{\tau} T+\frac{N}{\tau}$,

$K\left(\frac{\partial^{2} \phi}{\partial r^{2}}+\frac{2}{r} \frac{\partial \phi}{\partial r}\right)+\frac{E_{g}}{\tau} N=\left(1+\tau_{o} \frac{\partial}{\partial t}\right)\left(\rho c_{e} \frac{\partial T}{\partial t}+\gamma_{t} T_{o} \frac{\partial}{\partial t}\left(\frac{\partial u}{\partial r}+\frac{2 u}{r}\right)\right)$,

$\frac{\partial^{2} T}{\partial t^{2}}=\frac{\partial^{2} \phi}{\partial t^{2}}-a\left(\frac{\partial^{2} \phi}{\partial r^{2}}+\frac{2}{r} \frac{\partial \phi}{\partial r}\right)$,

with

$\sigma_{r r}=(\lambda+2 \mu) \frac{\partial u}{\partial r}+\lambda \frac{2 u}{r}-\gamma_{t} T-\gamma_{n} N$. 
$\sigma_{\theta \theta}=\sigma_{\phi \phi}=(\lambda+2 \mu) \frac{u}{r}+\lambda\left(\frac{\partial u}{\partial r}+\frac{u}{r}\right)-\gamma_{t} T-\gamma_{n} N$

\section{Application}

The initial conditions are supposed to be homogeneous. The bounding internal surface of cavity have the boundary conditions by the following

$$
\begin{aligned}
& u(R, t)=0, \\
& -\left.K \frac{\partial \phi(r, t)}{\partial r}\right|_{r=R}=q_{o} \frac{t^{2} e^{-\frac{t}{t_{p}}}}{16 t_{p}^{2}}, \\
& \left.D_{e} \frac{\partial N(r, t)}{\partial r}\right|_{r=R}=s_{O} N(R, t),
\end{aligned}
$$

To get main fields in dimensionless form, the following non-dimension variables can be used

$$
\begin{aligned}
& \left(r^{\circ}, u^{\circ}\right)=\eta c(r, u),\left(\sigma_{r r}^{\circ}, \sigma_{\theta \theta}^{\circ}\right)=\frac{\left(\sigma_{r r}, \sigma_{\theta \theta}\right)}{\lambda+2 \mu}, q_{o}^{\circ}=\frac{q_{o}}{\eta c T_{o} K}, a^{\circ}=\frac{a}{c^{2}}, \\
& \left(t^{\circ}, \tau^{\circ}, \tau_{o}^{\circ}, t_{p}^{\circ}\right)=\eta c^{2}\left(t, \tau, \tau_{o}, t_{p}\right), N^{\circ}=\frac{N}{n_{o}}, \phi^{\circ}=\frac{\phi}{T_{o}}, T^{\circ}=\frac{T}{T_{o}},
\end{aligned}
$$

where $\eta=\frac{\rho c_{e}}{K}$ and $c^{2}=\frac{\lambda+2 \mu}{\rho}$.

Using equation (15) for the main governing equations (dropping the superscript ${ }^{\circ}$ ), yields:

$$
\begin{aligned}
& \frac{\partial^{2} u}{\partial r^{2}}+\frac{2}{r} \frac{\partial u}{\partial r}-\frac{2 u}{r^{2}}-d_{1} \frac{\partial T}{\partial r}-d_{2} \frac{\partial N}{\partial r}=\frac{\partial^{2} u}{\partial t^{2}}, \\
& \frac{\partial^{2} N}{\partial r^{2}}+\frac{2}{r} \frac{\partial N}{\partial r}=d_{3} \frac{\partial N}{\partial t}+\frac{d_{3}}{\tau} N-\frac{d_{4}}{\tau} T, \\
& \frac{\partial^{2} \phi}{\partial r^{2}}+\frac{2}{r} \frac{\partial \phi}{\partial r}=-\frac{d_{5}}{\tau} N+\left(1+\tau_{o} \frac{\partial}{\partial t}\right)\left(\frac{\partial T}{\partial t}+d_{6} \frac{\partial}{\partial t}\left(\frac{\partial u}{\partial r}+\frac{2 u}{r}\right)\right), \\
& \frac{\partial^{2} \phi}{\partial t^{2}}=\frac{\partial^{2} T}{\partial t^{2}}+a\left(\frac{\partial^{2} \phi}{\partial r^{2}}+\frac{2}{r} \frac{\partial \phi}{\partial r}\right), \\
& \sigma_{r r}=\frac{\partial u}{\partial r}+d_{7} \frac{2 u}{r}-d_{1} T-d_{2} N, \\
& \sigma_{\theta \theta}=d_{7} \frac{\partial u}{\partial r}+\left(1+d_{7}\right) \frac{u}{r}-d_{1} T-d_{2} N, \\
& u(R, t)=0,\left.\frac{\partial \phi(r, t)}{\partial r}\right|_{r=R}=-q_{o} \frac{t^{2} e^{-\frac{t}{t_{p}}}}{16 t_{p}^{2}},\left.\frac{\partial N(r, t)}{\partial r}\right|_{r=R}=d_{8} N(R, t),
\end{aligned}
$$

where $d_{1}=\frac{T_{o} \gamma_{t}}{\lambda+2 \mu}, d_{2}=\frac{n_{o} \gamma_{n}}{\lambda+2 \mu}, d_{3}=\frac{1}{\eta D_{e}}, d_{4}=\frac{k T_{o}}{n_{o} \eta D_{e}}, d_{5}=\frac{n_{o} E_{g}}{\rho c_{e} T_{o}}$,

$$
d_{6}=\frac{\gamma_{t}}{\rho c_{e}}, d_{7}=\frac{\lambda}{\lambda+2 \mu}, d_{8}=\frac{S_{o}}{\eta c D_{e}} \text {. }
$$




\section{Laplace transform}

For $\mathrm{G}(r, t)$ function Laplace transform is defined by

$\overline{\mathrm{G}}(r, s)=L[\mathrm{G}(r, t)]=\int_{0}^{\infty} \mathrm{G}(r, t) e^{-s t} d t$.

Hence, the governing equations can be rewritten by

$$
\begin{aligned}
& \frac{d^{2} \bar{u}}{d r^{2}}+\frac{2}{r} \frac{d \bar{u}}{d r}-\frac{2 \bar{u}}{r^{2}}-d_{1} \frac{d \bar{T}}{d r}-d_{2} \frac{d \bar{N}}{d r}=s^{2} \bar{u}, \\
& \frac{d^{2} \bar{N}}{d r^{2}}+\frac{2}{r} \frac{d \bar{N}}{d r}=d_{3}\left(s+\frac{1}{\tau}\right) \bar{N}-\frac{d_{4}}{\tau} \bar{T}, \\
& \frac{d^{2} \bar{\phi}}{d r^{2}}+\frac{2}{r} \frac{d \bar{\phi}}{d r}=-\frac{d}{\tau} \bar{N}+\left(1+s \tau_{o}\right)\left(s \bar{T}+s d_{6}\left(\frac{d \bar{u}}{d r}+\frac{2 \bar{u}}{r}\right)\right), \\
& \bar{\phi}=\bar{T}+\frac{a}{s^{2}}\left(\frac{d^{2} \bar{\phi}}{d r^{2}}+\frac{2}{r} \frac{d \bar{\phi}}{d r}\right), \\
& \bar{\sigma}_{r r}=\frac{d \bar{u}}{d r}+d_{7} \frac{2 \bar{u}}{r}-d_{1} \bar{T}-d_{2} \bar{N}, \\
& \bar{\sigma}_{\theta \theta}=d_{7} \frac{d \bar{u}}{d r}+\left(1+d_{7}\right) \frac{\bar{u}}{r}-d_{1} \bar{T}-d_{2} \bar{N}, \\
& \bar{u}(R, t)=0,\left.\frac{d \bar{\phi}(r, t)}{d r}\right|_{r=R}=\frac{-q_{o} t_{p}}{8\left(s t_{p}+1\right)^{3}},\left.\frac{d \bar{N}(r, t)}{d r}\right|_{r=R}=d_{8} \bar{N}(R, t),
\end{aligned}
$$

Differentiating equations (24), (25) and (26) with respect to $r$ and using equation (27), yields:

$\frac{d^{2} \bar{u}}{d r^{2}}+\frac{2}{r} \frac{d \bar{u}}{d r}-\frac{2 \bar{u}}{r^{2}}=x_{11} \bar{u}+x_{12} \frac{d \bar{N}}{d r}+x_{13} \frac{d \bar{\phi}}{d r}$

$\frac{d^{2}}{d r^{2}}\left(\frac{d \bar{N}}{d r}\right)+\frac{2}{r} \frac{d}{d r}\left(\frac{d \bar{N}}{d r}\right)-\frac{2}{r^{2}}\left(\frac{d \bar{N}}{d r}\right)=x_{21} \bar{u}+x_{22} \frac{d \bar{N}}{d r}+x_{23} \frac{d \bar{\phi}}{d r}$,

$\frac{d^{2}}{d r^{2}}\left(\frac{d \bar{\phi}}{d r}\right)+\frac{2}{r} \frac{d}{d r}\left(\frac{d \bar{\phi}}{d r}\right)-\frac{2}{r^{2}}\left(\frac{d \bar{\phi}}{d r}\right)=x_{31} \bar{u}+x_{32} \frac{d \bar{N}}{d r}+x_{33} \frac{d \bar{\phi}}{d r}$

where

$$
\begin{aligned}
& x_{11}=\left(s^{2}-d_{1} x_{31} \frac{a}{s^{2}}\right), x_{12}=\left(d_{2}-d_{1} x_{32} \frac{a}{s^{2}}\right), x_{13}=d_{1}\left(1-\frac{a}{s^{2}} x_{33}\right) \\
& x_{21}=\frac{d_{4}}{\tau} \frac{a}{s^{2}} x_{31}, x_{22}=\left(d_{3}\left(s+\frac{1}{\tau}\right)+\frac{d_{4}}{\tau} \frac{a}{s^{2}} x_{32}\right), x_{23}=\frac{d_{4}}{\tau}\left(\frac{a}{s^{2}} x_{33}-1\right) \\
& x_{31}=\frac{d_{6} s^{3}\left(1+s \tau_{o}\right)}{\left(1+s\left(1+s \tau_{o}\right)\left(1+d_{6} d_{1}\right) \frac{a}{s^{2}}\right)}, x_{32}=\frac{\left(d_{2} s\left(1+s \tau_{o}\right) d_{6}-\frac{d_{5}}{\tau}\right)}{\left(1+s\left(1+s \tau_{o}\right)\left(1+d_{6} d_{1}\right) \frac{a}{s^{2}}\right)}, x_{33}=\frac{s\left(1+s \tau_{o}\right)\left(1+d_{6} d_{1}\right)}{\left(1+s\left(1+s \tau_{o}\right)\left(1+d_{6} d_{1}\right) \frac{a}{s^{2}}\right)} .
\end{aligned}
$$

Now, it is possible to obtain the solutions of the equations (31), (32) and (33) by the eigenvalue approach proposed [39-44]. The vectors-matrix of equations (31-33) can be given by $D V=X V$,

where $D=\frac{d^{2}}{d r^{2}}+\frac{2}{r} \frac{d}{d r}-\frac{2}{r^{2}}, \mathrm{~V}=\left[\begin{array}{lll}\bar{u} & \frac{d \bar{N}}{d r} & \frac{d \Phi}{d r}\end{array}\right]^{T}$ and $X=\left[\begin{array}{lll}x_{11} & x_{12} & x_{13} \\ x_{21} & x_{22} & x_{23} \\ x_{31} & x_{32} & x_{33}\end{array}\right]$. 
The characteristic formulation of matrix $X$ are defined by

$m^{3}-m^{2}\left(x_{11}+x_{22}+x_{33}\right)+m\left(-x_{22} x_{33}+x_{11} x_{22}+x_{11} x_{33}-x_{21} x_{12}-x_{13} x_{31}\right)+$

$x_{12} x_{23} x_{32}-x_{12} x_{22} x_{33}-x_{12} x_{23} x_{31}-x_{13} x_{21} x_{32}+x_{13} x_{22} x_{31}+x_{12} x_{21} x_{33}=0$,

The eigenvalues of matrix $X$ are the three roots of equation (35) which define by the form $m_{1}, m_{2}, m_{3}$. Thus, the corresponding eigenvector $Y$ can be determined as:

$Y=\left(\begin{array}{c}\left(x_{22}-m\right) x_{13}-x_{12} x_{23} \\ x_{23}\left(x_{11}-m\right)-x_{13} x_{21} \\ x_{22}\left(m-x_{11}\right)+x_{12} x_{21}+m x_{11}-m^{2}\end{array}\right)$

The solutions of equations (34) which are bounded as $r \rightarrow \infty$ can be given by

$\mathrm{V}(r, s)=\sum_{i=1}^{3} B_{i} X_{i} r^{-1 / 2} K_{3 / 2}\left(s_{i} r\right)$

where $K_{3 / 2}$ is the modified of Bessel's function of order $\frac{3}{2}$ and $n_{i}=\sqrt{m_{i}}, B_{1}, B_{2}$ and $B_{3}$ are constants can be coputed by using the boundary conditions of problem. Thus, the variables solutions along $r$ and $s$ can be expressed by

$\bar{u}(r, s)=\sum_{i=1}^{3} A_{i} U_{i} r^{-1 / 2} K_{3 / 2}\left(n_{i} r\right)$

$\bar{N}(r, s)=-\sum_{i=1}^{3} A_{i} N_{i} r^{-1 / 2} K_{1 / 2}\left(n_{i} r\right)$

$\bar{\phi}(r, s)=-\sum_{i=1}^{3} A_{i} T_{i} r^{-1 / 2} K_{1 / 2}\left(n_{i} r\right)$,

$\bar{T}(r, s)=-\sum_{i=1}^{3} A_{i} \frac{T_{i}\left(1-\frac{a}{s^{2}} n_{i}^{2}\right)}{r^{1 / 2} n_{i}} K_{1 / 2}\left(n_{i} r\right)$,

Finally, Stehfest [45] numerical inversion method has been chosen as in [46] to get the numerical inversions of physical quantities.

\section{Numerical Results and Discussions}

To theoretically study the results obtained, the physical properties and physical constants of silicon ( $\mathrm{Si}$ ) as an elastic semiconductor material are used. The constants of silicon ( $\mathrm{Si}$ ) are used to do the numerical simulation and discussed the calculation results, the constants of Si can be given by [47]:

$\lambda=3.64 \times 10^{10}(\mathrm{~N})\left(\mathrm{m}^{-2}\right), \tau=5 \times 10^{-5}(\mathrm{~s}), n_{o}=10^{20}\left(\mathrm{~m}^{-3}\right), s_{o}=2(\mathrm{~m})\left(\mathrm{s}^{-1}\right), T_{o}=300(\mathrm{k})$, $\mu=5.46 \times 10^{10}(N)\left(m^{-2}\right), \alpha_{t}=3 \times 10^{-6}\left(k^{-1}\right), d_{n}=-9 \times 10^{-31}\left(m^{3}\right), t_{p}=0.5, \tau_{o}=0.05$, 
$\rho=2330(\mathrm{~kg})\left(\mathrm{m}^{-3}\right), c_{e}=695(\mathrm{~J})\left(\mathrm{kg}^{-1}\right)\left(\mathrm{k}^{-1}\right), E_{g}=1.11(\mathrm{eV}), D_{e}=2.510^{-3}\left(\mathrm{~m}^{2}\right)\left(\mathrm{s}^{-1}\right)$.

Based on the above set of parameters, the calculations of physical variables (numerically) along the redial distance $r$ for the generalized hyperbolic two-temperatures model and the classical twotemperatures theory are presented in figures 1-6. The numerical computational are carry out when using the above set of constants of field distributions of the basic quantities as (thermal waves (thermo-dynamical temperature distributions), the redial displacement distribution (strain wave), the redial and hoop stress which describe the mechanical waves distributions, the carrier density distribution (plasma waves). Figures 1 explain the variations of carrier density with respect to the radial distancee $r$. It is clear that it begin with its maxxmum values at the boundary $r=R$ then it gradually decrease with the increasing of $r$ until it reach to zeros. Figure 2 show the thermodynamic temperature variation with respect to the redial distance $r$. It is observed that the thermodynamic temperature begins from the maximums values at the boundary $r=R$ and reduces with the rising of the redial distancce $r$ until it comes to zero value. Figure 3 display the conductive temperature variations along the redial distance $r$. It is observed that the it has maxmum values at the boundary $r=R$ after that it decreases gradually with the increasing of $r$ to reach to zero. Figures 4 desplay the variation of radial displacement with respect to the redial distance $r$. It observed that it starts from zero values which satisfy the boundary conditions then the radial displacement progressively increases up to peak values and then decreases again to reach to zeros. Figures 5 and 6 show the variations of radial and hoop stresses along the radial distancee $r$. It clear that the magnitudes of stresses started from the maximum values at the surface of cavity after that decreases rapidly as $r$ increases to reach zero values. Finally, in compressions between the solutions, one can conclude that considering new hyperbolic two-temperatures photothermoelastic model have an important effect on the distributions of field quantities.

\section{Acknowledgment}

This project was funded by the Deanship of Scientific Research (DSR) at King Abdulaziz University, Jeddah, Saudi Arabia, under grant no. (KEP-77-130-42). The authors, therefore, acknowledge with thanks DSR technical and financial support.

\section{Availability of Data and Material}

There is no data or material that has been copied from elsewhere in the proposed manuscript.

\section{Author Contributions}


The authors have equal contribution in the paper.

\section{Funding}

This project was funded by the Deanship of Scientific Research (DSR) at King Abdulaziz

University, Jeddah, Saudi Arabia, under grant no. (KEP-77-130-42).

\section{Declarations}

The authors declare that the manuscript follows ethical standards as per the guidelines provided during manuscript submission.

\section{Conflict of Interest}

The authors declare that there is no conflict of interest in the proposed manuscript as far as the publication is concerned.

\section{Consent to Participate}

There is mutual understanding between the two authors and is a combined work.

\section{Consent for Publication}

The authors have full faith on the publisher hence the publisher has full right for publication as per their guidelines.

\section{References}

1. Biot, MA (1956) Thermoelasticity and irreversible thermodynamics. Journal of applied physics, 27(3): 240-253.

2. Lord, HW, Shulman, Y (1967) A generalized dynamical theory of thermoelasticity. Journal of the Mechanics and Physics of Solids, 15(5): 299-309.

3. Chen, PJ, Gurtin, ME, Williams, WO (1969) On the thermodynamics of non-simple elastic materials with two temperatures. Zeitschrift für angewandte Mathematik und Physik ZAMP, 20(1): 107-112.

4. Chen, PJ, Gurtin, ME (1968) On a theory of heat conduction involving two temperatures. Zeitschrift für angewandte Mathematik und Physik (ZAMP), 19(4): 614-627.

5. Gurtin, ME, Williams, WO (1967) An axiomatic foundation for continuum thermodynamics. Archive for Rational Mechanics and Analysis, 26(2): 83-117.

6. Youssef, HM, El-Bary, AA (2018) THEORY OF HYPERBOLIC TWO-TEMPERATURE GENERALIZED THERMOELASTICITY. Materials Physics and Mechanics, 40): 158-171.

7. Taye, IM, Lotfy, K, El-Bary, A, Alebraheem, J, Asad, S (2021) The Hyperbolic Two Temperature Semiconducting Thermoelastic Waves by Laser Pulses. CMC-COMPUTERS MATERIALS \& CONTINUA, 67(3): 3601-3618.

8. Saeed, T, Abbas, I (2020) A hyperbolic two-temperature photo-thermal interactions in a semiconductor material. Indian Journal of Physics): 1-6. 
9. Abbas, I, Saeed, T, Alhothuali, M (2020) Hyperbolic Two-Temperature Photo-Thermal Interaction in a Semiconductor Medium with a Cylindrical Cavity. Silicon): 1-8.

10. Lotfy, K, El-Bary, A, Tantawi, R (2019) Effects of variable thermal conductivity of a small semiconductor cavity through the fractional order heat-magneto-photothermal theory. The European Physical Journal Plus, 134(6): 280.

11. Lotfy, K, Hassan, W, El-Bary, A, Kadry, MA (2020) Response of electromagnetic and Thomson effect of semiconductor medium due to laser pulses and thermal memories during photothermal excitation. Results in Physics, 16): 102877.

12. Hobiny, A, Abbas, I (2019) A GN model on photothermal interactions in a two-dimensions semiconductor half space. Results in Physics, 15): 102588.

13. Ali, H, Jahangir, A, Khan, A (2019) Reflection of waves in a rotating semiconductor nanostructure medium through torsion-free boundary condition. Indian Journal of Physics): 1-9.

14. Yasein, Md, Mabrouk, N, Lotfy, K, EL-Bary, A (2019) The influence of variable thermal conductivity of semiconductor elastic medium during photothermal excitation subjected to thermal ramp type. Results in Physics, 15): 102766.

15. Lotfy, K, El-Bary, A, Hassan, W, Alharbi, A, Almatrafi, M (2020) Electromagnetic and Thomson effects during photothermal transport process of a rotator semiconductor medium under hydrostatic initial stress. Results in Physics, 16): 102983.

16. Alzahrani, FS, Abbas, IA (2019) Photo-thermo-elastic interactions without energy dissipation in a semiconductor half-space. Results in Physics, 15): 102805.

17. Abbas, I, Hobiny, AD (2021) Analytical-numerical solutions of photo-thermal interactions in semiconductor materials. Information Sciences Letters, 10(2): 189-196.

18. Youssef, HM, El-Bary, AA (2021) Characterization of the Photothermal Interaction of a Semiconducting Solid Sphere Due to the Fractional Deformation, Relaxation Time, and Various Reference Temperature under L-S Theory. Silicon, 13(7): 2103-2114.

19. Lotfy, K, Elidy, ES, Tantawi, RS (2021) Photothermal Excitation Process during Hyperbolic TwoTemperature Theory for Magneto-Thermo-Elastic Semiconducting Medium. Silicon, 13(7): 22752288.

20. Hobiny, AD, Abbas, IA (2017) A study on photothermal waves in an unbounded semiconductor medium with cylindrical cavity. Mechanics of Time-Dependent Materials, 21(1): 61-72.

21. Bera, MB, Das, NC, Lahiri, A (2015) Eigenvalue approach to two-temperature generalized thermoelastic interactions in an annular disk. Journal of Thermal Stresses, 38(11): 1310-1324.

22. Santra, S, Lahiri, A, Das, NC (2014) Eigenvalue approach on thermoelastic interactions in an infinite elastic solid with voids. Journal of Thermal Stresses, 37(4): 440-454.

23. Lata, P, Kaur, I (2018) Effect of hall current in Transversely Isotropic magneto thermoelastic rotating medium with fractional order heat transfer due to normal force. Advances in materials Research, 7(3): 203-220.

24. Sarkar, N, Mondal, S (2019) Two-dimensional problem of two-temperature generalized thermoelasticity using memory-dependent heat transfer: an integral transform approach. Indian Journal of Physics): 1-10.

25. Sur, A, Mondal, S (2021) The Caputo-Fabrizio heat transport law in vibration analysis of a microscale beam induced by laser. ZAMM-Journal of Applied Mathematics and Mechanics/Zeitschrift für Angewandte Mathematik und Mechanik, 101(3): e202000215.

26. Sarkar, N (2020) Thermoelastic responses of a nonlocal elastic rod due to nonlocal heat conduction. ZAMM-Journal of Applied Mathematics and Mechanics/Zeitschrift für Angewandte Mathematik und Mechanik, 100(4): e201900252.

27. Alharbi, AM, Abd-Elaziz, EM, Othman, MI (2020) Effect of temperature-dependent and internal heat source on a micropolar thermoelastic medium with voids under 3PHL model. ZAMM-Journal 
of Applied Mathematics and Mechanics/Zeitschrift für Angewandte Mathematik und Mechanik): e202000185.

28. Sarkar, N, Bachher, M, Das, N, De, S, Sarkar, N (2020) Waves in nonlocal thermoelastic solids of type III. ZAMM-Journal of Applied Mathematics and Mechanics/Zeitschrift für Angewandte Mathematik und Mechanik, 100(4): e201900074.

29. Sarkar, N, Mondal, S (2020) Thermoelastic plane waves under the modified Green-Lindsay model with two-temperature formulation. ZAMM-Journal of Applied Mathematics and Mechanics/Zeitschrift für Angewandte Mathematik und Mechanik, 100(11): e201900267.

30. Saeed, T, Abbas, I, Marin, M (2020) A gl model on thermo-elastic interaction in a poroelastic material using finite element method. Symmetry, 12(3): 488.

31. Marin, M, Vlase, S, Paun, M (2015) Considerations on double porosity structure for micropolar bodies. Aip Advances, 5(3): 037113.

32. Marin, M (1999) An evolutionary equation in thermoelasticity of dipolar bodies. Journal of Mathematical Physics, 40(3): 1391-1399.

33. Palani, G, Abbas, I (2009) Free convection MHD flow with thermal radiation from an impulsivelystarted vertical plate. Nonlinear Analysis: Modelling and Control, 14(1): 73-84.

34. Abbas, IA, Aly, KA, Dahshan, A (2018) Analytical Solutions of Plasma and Thermoelastic Waves Photogenerated by a Focused Laser Beam in a Semiconductor Material. Silicon, 10(6): 2609-2616.

35. Hobiny, A, Abbas, I (2020) Fractional Order GN Model on Photo-Thermal Interaction in a Semiconductor Plane. Silicon, 12(8): 1957-1964.

36. Alzahrani, FS, Abbas, IA (2021) A Study of Photo-Thermoelastic Wave in Semiconductor Materials with Spherical Holes Using Analytical-Numerical Methods. Silicon.

37. Song, Y, Bai, J, Ren, Z (2012) Study on the reflection of photothermal waves in a semiconducting medium under generalized thermoelastic theory. Acta Mechanica, 223(7): 1545-1557.

38. Mandelis, A, Nestoros, M, Christofides, C (1997) Thermoelectronic-wave coupling in laser photothermal theory of semiconductors at elevated temperatures. Optical Engineering, 36(2): 459-468.

39. Das, NC, Lahiri, A, Giri, RR (1997) Eigenvalue approach to generalized thermoelasticity. Indian Journal of Pure and Applied Mathematics, 28(12): 1573-1594.

40. Abbas, IA (2014) Eigenvalue approach in a three-dimensional generalized thermoelastic interactions with temperature-dependent material properties. Computers \& Mathematics with Applications, 68(12): 2036-2056.

41. Abbas, IA (2014) Eigenvalue approach for an unbounded medium with a spherical cavity based upon two-temperature generalized thermoelastic theory. Journal of Mechanical Science and Technology, 28(10): 4193-4198.

42. Abbas, IA (2015) A dual phase lag model on thermoelastic interaction in an infinite fiberreinforced anisotropic medium with a circular hole. Mechanics Based Design of Structures and Machines, 43(4): 501-513.

43. Abbas, IA (2015) The effects of relaxation times and a moving heat source on a two-temperature generalized thermoelastic thin slim strip. Canadian Journal of Physics, 93(5): 585-590.

44. Lahiri, A, Das, B, Sarkar, S. Eigenvalue approach to thermoelastic interactions in an unbounded body with a spherical cavity. in Proceedings of the World Congress on Engineering. 2010.

45. Stehfest, H (1970) Algorithm 368: Numerical inversion of Laplace transforms [D5]. Communications of the ACM, 13(1): 47-49.

46. Alzahrani, FS, Abbas, IA (2020) Photo-thermal interactions in a semiconducting media with a spherical cavity under hyperbolic two-temperature model. Mathematics, 8(4): 585. 
47. Song, Y, Todorovic, DM, Cretin, B, Vairac, P, Xu, J, Bai, J (2014) Bending of Semiconducting Cantilevers Under Photothermal Excitation. International Journal of Thermophysics, 35(2): 305319.

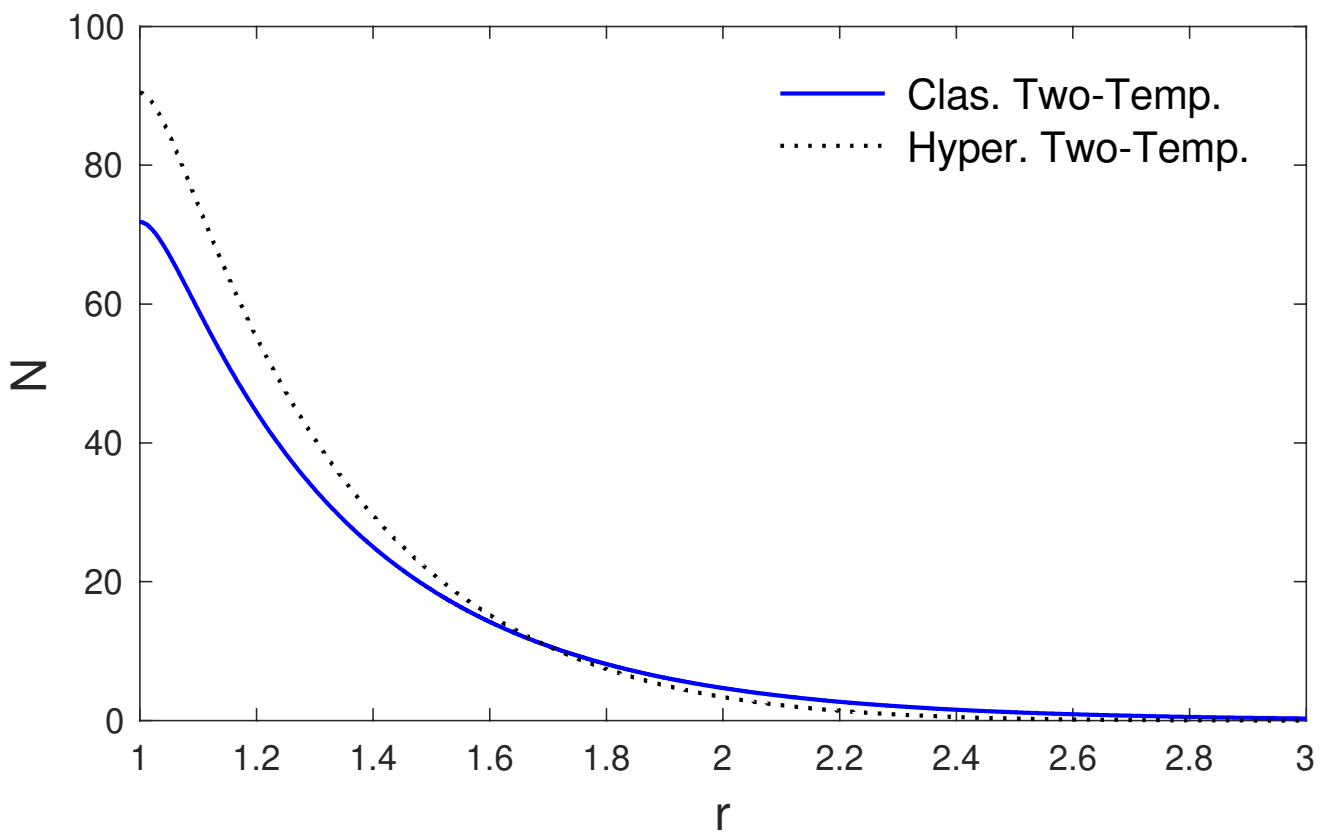

Fig. 1 The carrier density variations versus the redial distance.

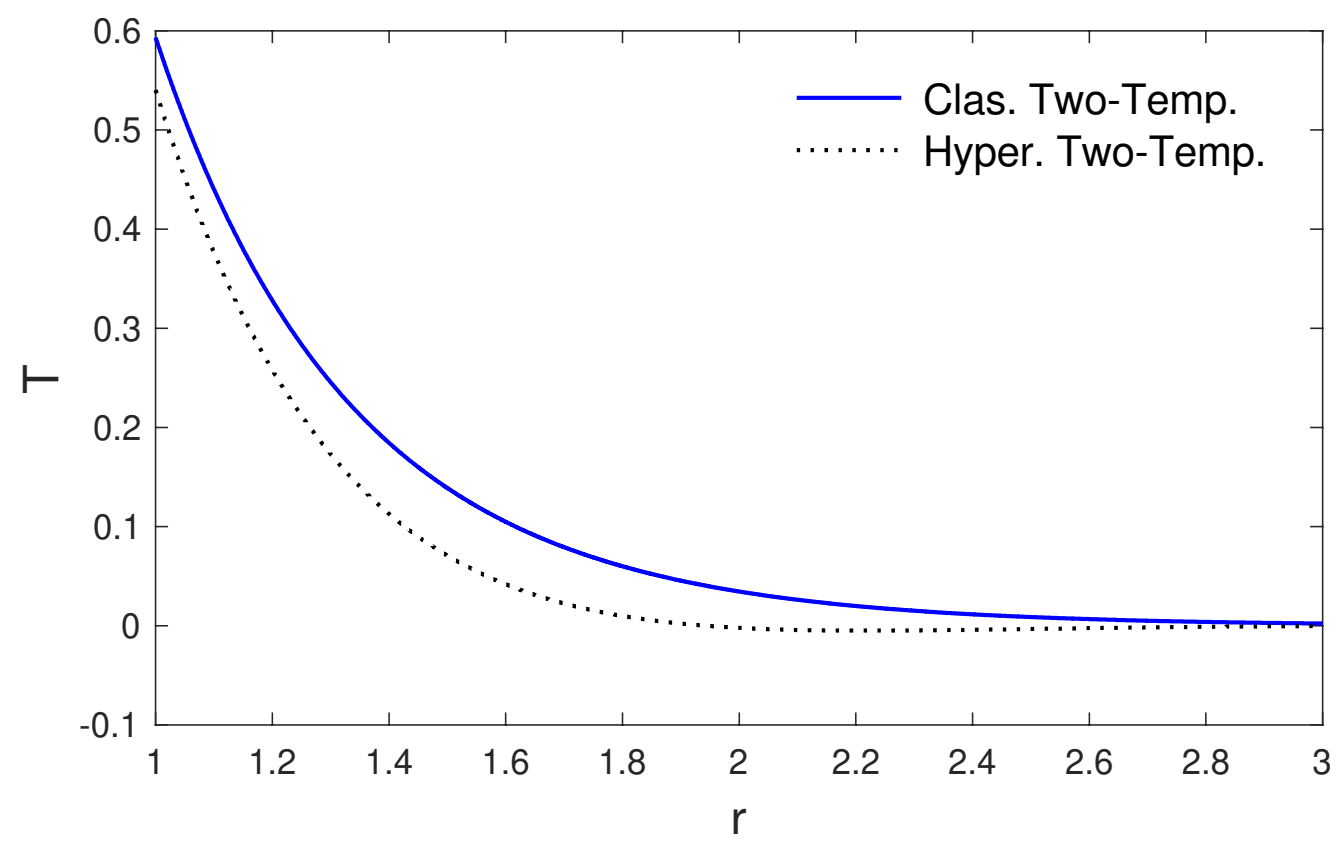

Fig. 2 The thermodynamic temperature variations versus the redial distance. 


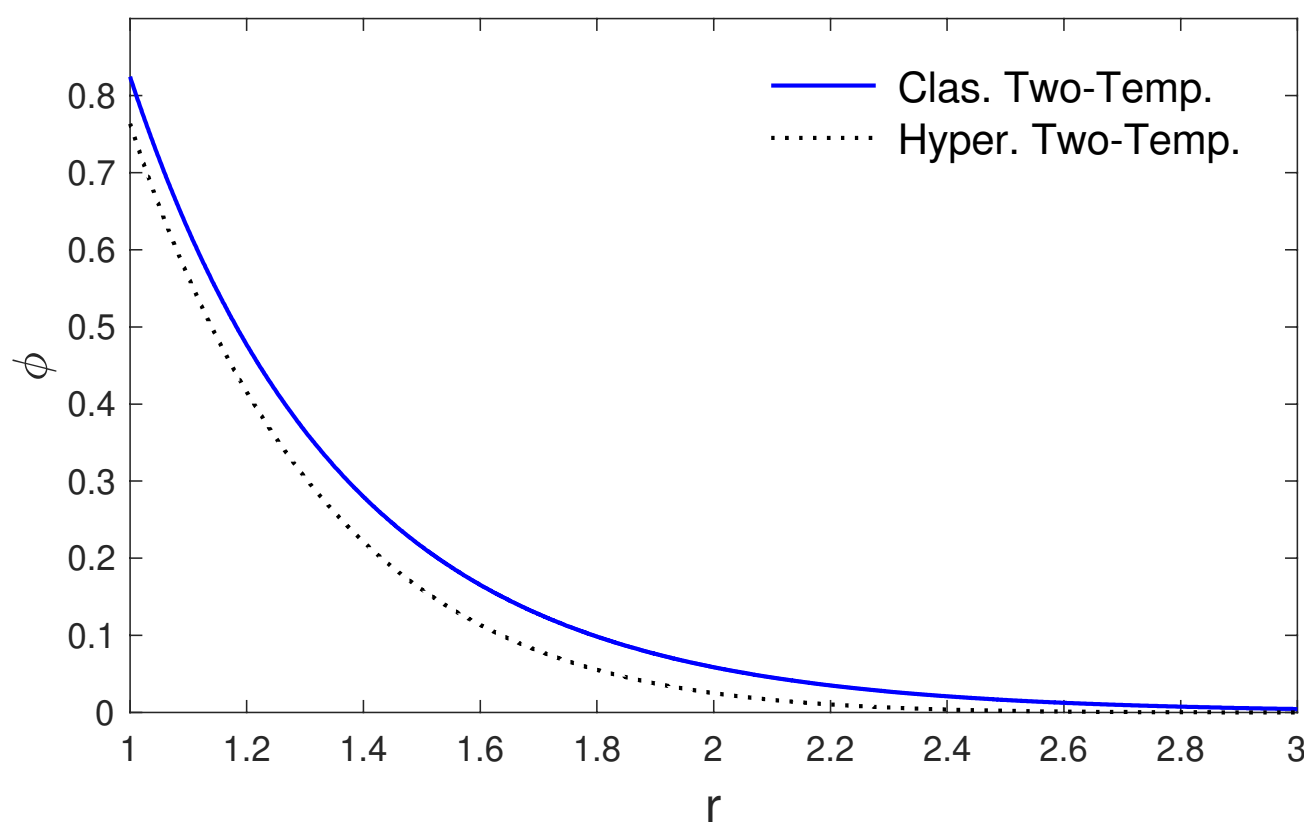

Fig. 3 The conductive temperature variations versus the redial distance.

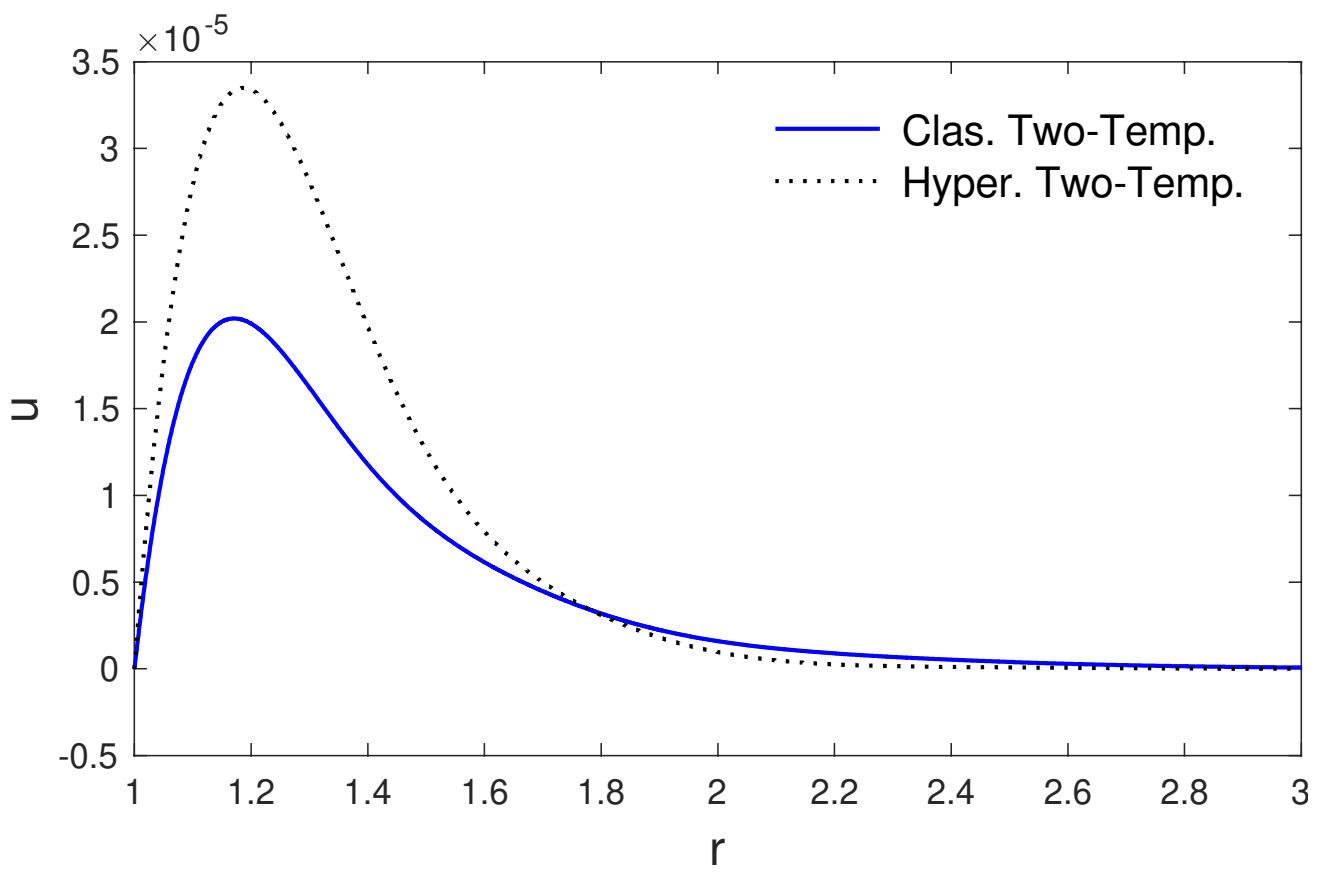

Fig. 4 The displacement variations versus the redial distance. 


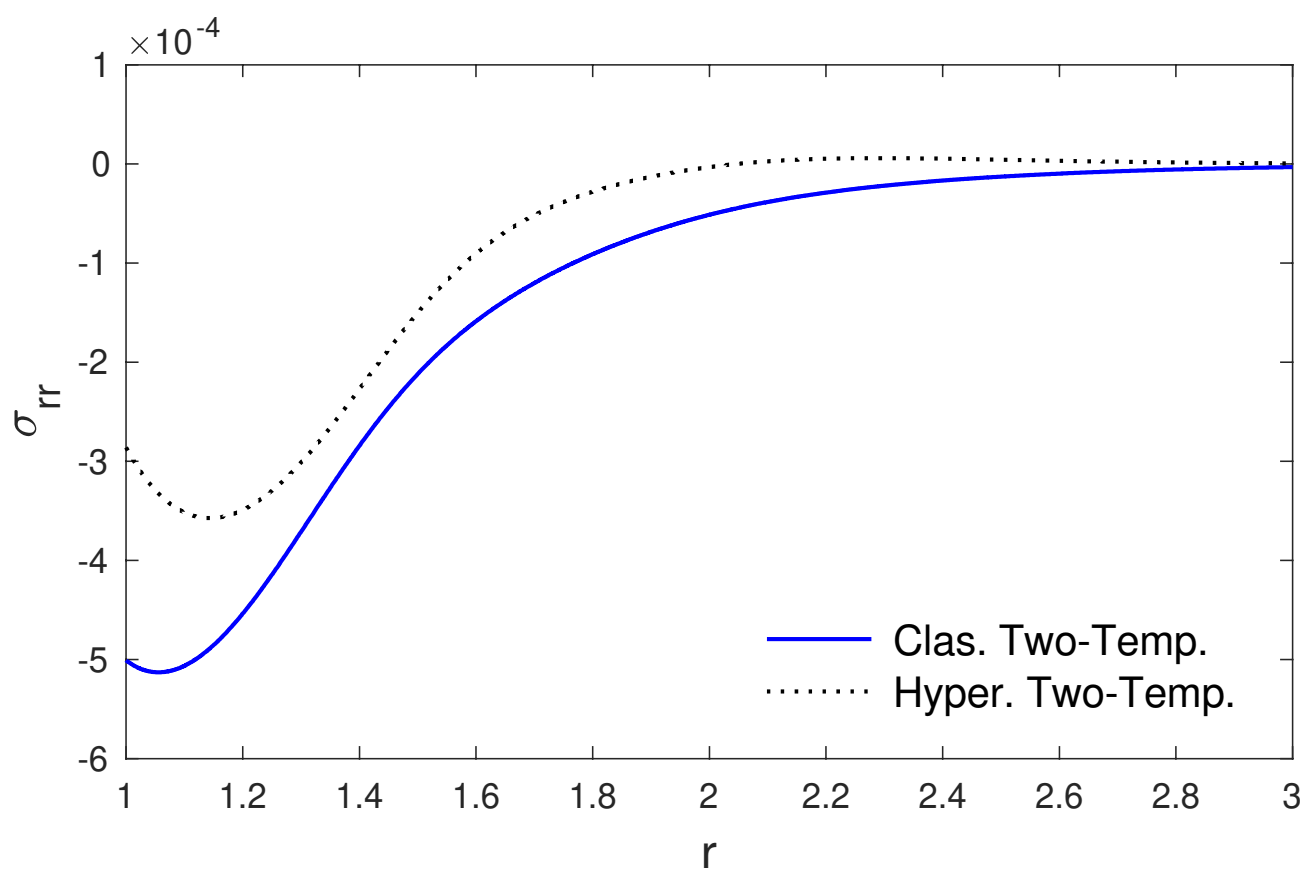

Fig. 5 The radial stress variations versus the redial distance.

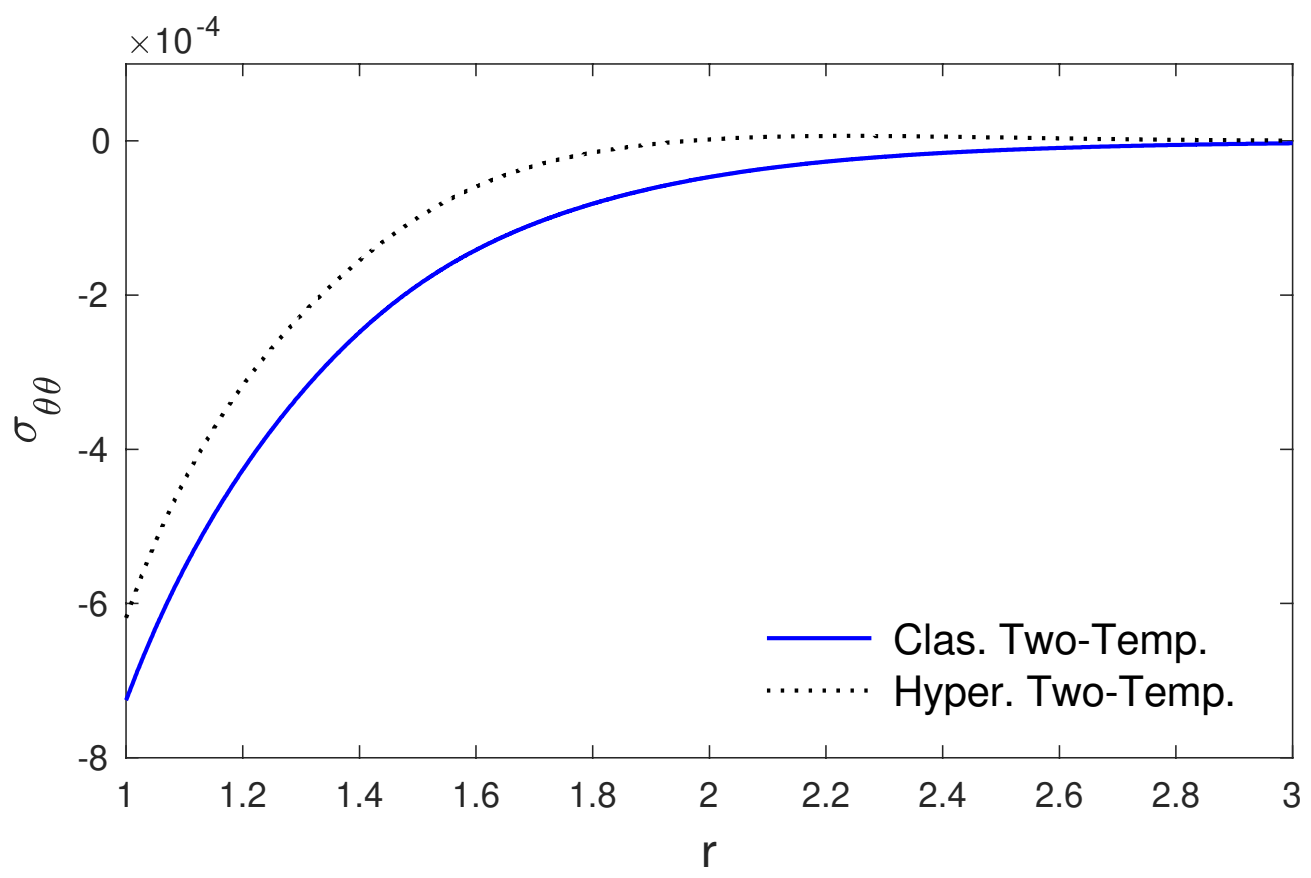

Fig. 6 The hoop stress variations versus the redial distance. 